\title{
METONIMIA COMO RECURSO COHESIONADOR EN EL TEXTO PERIODÍSTICO DEPORTIVO
}

\author{
METONYMY AS A COHESION TOOL IN SPORTS NEWSPAPER ARTICLES
}

\author{
SARA Quintero RAMíreZ \\ Universidad de Guadalajara \\ qsara@hotmail.com
}

Recibido: $17 / 08 / 2017$

Aceptado: 25/09/2017

\begin{abstract}
Resumen
En este artículo nos proponemos identificar las metonimias más comunes en un corpus de 150 notas periodísticas deportivas en español, francés e inglés. Para lograr lo anterior, primero determinamos el tipo de metonimias que se utilizan en las tres lenguas que nos ocupan y posteriormente, distinguimos el tipo de metonimias que solamente se utilizan en una lengua en particular. Con lo anterior, pretendemos establecer la función textual de las metonimias en el marco de la nota periodística deportiva. Con base en la observación de nuestros materiales, hemos inventariado una diversidad de metonimias que van desde términos hiperónimos hasta sintagmas nominales elaborados que proporcionan información específica de un equipo o atleta. Por último, advertimos que las metonimias son recursos textuales anafóricos de los que se sirve el periodista deportivo para designar a los diferentes referentes de sus notas en innumerables ocasiones y conferirle unidad a su texto.

PALABRAS CLAVE: metonimias, discurso deportivo, cohesión textual, recurso anafórico.
\end{abstract}

\begin{abstract}
The purpose of this study is to identify the metonymies that are most commonly used in a newspaper corpus of 150 sports articles written in Spanish, French and English. In order to do this, we first of all determine the metonymies that are used in the three languages and secondly, we distinguish those metonymies that are only used in one language. The importance of this study lies in the identification of the textual function of metonymies in sports newspaper articles. Based on the corpus analysis, a diversity of metonymies is inventoried, from those that take advantage of hypernyms to those that use elaborated noun phrases that offer specific information regarding a sports team or an athlete. Finally, we conclude that metonymies are anaphoric textual resources that sports journalists use in order to name the different referents in their articles and give unity to their texts.
\end{abstract}

KEYWORDS: metonymies, sports discourse, textual cohesion, anaphoric resource.

Para citar este artículo / To cite this article: Quintero Ramírez, Sara (2017). Metonimia como recurso cohesionador en el texto periodístico deportivo. ELUA, 31: 269-284. doi: 10.14198/ELUA2017.31.14

Enlace / Link: http://dx.doi.org/10.14198/ELUA2017.31.14 


\section{INTRODUCCIÓN}

El discurso periodístico deportivo se caracteriza, entre otros rasgos, por hacer uso constante de figuras retóricas tales como la metáfora, la metonimia, la sinécdoque, la hipérbole, el símil, la aliteración, entre otras. Tanto comentaristas de crónicas orales como periodistas de notas escritas se valen de estas figuras para la producción de sus textos (Cf. Nomdedeu Rull, 2004; Saiz Noeda, 2010; Corado Valenzuela, 2011; Oliva Marañón, 2012; Suárez Ramírez y Suarez Muñoz, 2016; Quintero Ramírez y Álvarez Amaral, 2016; etc.).

Un sinnúmero de estudios se ha encauzado en el empleo de la metáfora en el lenguaje deportivo (Cf. Segrave, 2000; Herráez Pindado, 2004; Silaški, 2009; Segura Soto, 2009; Medina Montero, 2007, 2009 y 2015). No obstante, son escasos aquellos trabajos que se han dedicado a la metonimia en este mismo discurso. En efecto, los pocos estudios que hemos encontrado respecto de la metonimia empleada en el texto deportivo también examinan la metáfora (Cf. Saiz Noeda, 2010; Quintero Ramírez y Álvarez Amaral, 2016; Suárez Ramírez y Suárez Muñoz, 2016) ${ }^{1}$.

En realidad, el fenómeno que referimos en el párrafo anterior se extiende al estudio de la metonimia en general, pues los pocos trabajos que hablan de metonimia lo hacen en un espacio reducido en el marco de estudios mucho más enfocados en la metáfora; tal es el caso del libro de Lakoff y Johnson (1980), así como el de Kövecses (2002). Con la excepción de obras como las de Denroche (2012 y 2015), que se centran en la metonimia.

En el presente estudio tenemos el objetivo de identificar las metonimias más utilizadas en un corpus constituido de 150 notas periodísticas deportivas en español, francés e inglés; esto es, 50 notas en cada idioma. Asimismo, nos proponemos determinar aquellas metonimias que resultan comunes en las tres lenguas antes aludidas, así como aquellas que son exclusivas de alguna lengua en particular. Por último, nos planteamos el propósito de distinguir la función textual que desempeñan las metonimias de dicho corpus.

Con la intención de presentar el estudio que hemos realizado, en primer lugar, nos valemos de un apartado con los fundamentos teóricos en donde explicamos a qué tipo de lenguaje pertenece el discurso deportivo, qué es la metonimia y cuáles son las metonimias que estudiosos del lenguaje del deporte han registrado en sus respectivas investigaciones. En seguida, mostramos una sección metodológica en la que elucidamos cómo conformamos el corpus de este estudio y cómo hemos conducido nuestro análisis. Posteriormente, revelamos nuestros resultados y los examinamos de acuerdo con las dilucidaciones de los fundamentos teóricos. Finalmente, damos lugar a las conclusiones a las que hemos llegado en este trabajo.

\section{FUNDAMENTOS TEÓRICOS}

\subsection{Discurso deportivo}

De acuerdo con Castañón Rodríguez (2012: 344), el deporte se ha vuelto un fenómeno que se ha expandido mundialmente. Dicha mundialización ha provocado que se

1 El propio Medina Montero (2007: 203), con base en elucidaciones de Ullmann (1991), advierte que su trabajo deja de lado la metonimia, porque esta, a diferencia de la metáfora, no manifiesta nuevas relaciones, sino que se origina de las relaciones ya establecidas entre las palabras. 
difunda una gran cantidad de información respecto de todo tipo de eventos deportivos. Esta difusión se torna cada vez más especializada y se conciben diversas estrategias de comunicación para un sinfín de objetivos tales como: a) proyectar una imagen favorable de las entidades deportivas al público aficionado; b) establecer ambientes propicios para el consumo de productos relacionados con dichas entidades (camisetas, calendarios, banderines, etc.); c) crear identificación y proximidad del aficionado con los deportistas (Vélez, 2010: 219).

Como mencionamos anteriormente, la difusión del deporte se ha vuelto tan especializada que el lenguaje deportivo es considerado un discurso que resulta accesible solamente a aquellos que están familiarizados con él (Saiz Noeda, 2010: 197; Gómez Torrego, 2010: 151). En efecto, Mapelli (2004: 171) advierte que, con el afán de adornar su discurso, el periodista se sirve de locuciones creativas que llegan a utilizarse tanto en el ámbito deportivo que luego forman parte de dicha tradición discursiva. Estas expresiones no son transparentes semánticamente y, por ende, le exigen a la audiencia un "esfuerzo de interpretación" (Mapelli, 2004: 173).

De acuerdo con Medina Montero (2007: 197-198), los lenguajes de especialidad se dividen en tres grandes grupos: a) lenguajes científico-técnicos; b) lenguajes sectoriales y c) argots. El discurso deportivo pertenece al lenguaje sectorial, porque dichos lenguajes suelen tener un elemento sociológico que se evidencia mediante los campos de las profesiones, así como de las actividades específicas. Igualmente, este tipo de discursos contribuye, como lo afirma Vélez (2010), a la identificación entre los diferentes individuos de una agrupación.

Oliva Marañón (2012) ratifica lo señalado por Medina Montero:

El desarrollo extraordinario de la información deportiva a ambos lados del Atlántico ha propiciado la creación de un lenguaje sectorial, con multitud de términos y expresiones nuevas, que ha evolucionado con personalidad propia en los últimos años hasta convertirse en terreno de avanzadilla de la lengua española (Oliva Marañón, 2012: 14).

En el marco del discurso periodístico publicado en prensa, Hernando Cuadrado (1994) y Yanes Mesa (2006) presentan diversos géneros, tales como: artículo, reportaje, editorial, columna, crónica, etc. Cabe mencionar que en nuestro estudio no nos enfocamos en ningún género periodístico en particular, sino que examinamos notas periodísticas deportivas en general. La nota periodística, como expone Martínez Valle (1997: 59), "es una relación creativa, a veces subjetiva, cuyo principal objeto es formar la opinión del público a través de la información de un suceso o de una situación".

El discurso periodístico deportivo, en especial aquel producido en prensa impresa y digital, constituye un objeto de estudio sumamente frecuente para lingüistas, justamente por la peculiaridad de sus términos y expresiones creativas que se actualizan continuamente (Alvar Ezquerra, 2009: 158-159; Arroyo Almaraz y García García, 2012: 318). De tal manera, el lenguaje deportivo cuenta con expresiones propias que han llegado a consolidarse y difundirse considerablemente. Entre dichas expresiones se encuentran las metonimias, fenómeno que elucidamos en el siguiente apartado. 


\subsection{Metonimia en el discurso deportivo}

El término metonimia significa trasnominación (Corado Valenzuela, 2011: 28). Se trata de una figura retórica que se encarga de designar una cosa mediante el nombre de otra. Dicha designación es motivada por relaciones de contigüidad o inclusión entre los significados de ambas (Sánchez Manzanares, 2008: 202).

Denroche advierte que la metonimia es una "etapa" o "sub-proceso" de la metáfora, por lo que la primera es más fundamental que la segunda. Este autor define la metonimia como "[...] the highlighting of relatedness, usually part-whole, between closely-related concepts, things and signifiers" (2015: 56).

Para Denroche (2015: 3), la metonimia se produce en un ámbito complejo, pues interviene en diferentes niveles de la lengua y la comunicación; esto es, desde el nivel de la palabra, hasta el nivel de las interacciones. Lo anterior implica que la metonimia actúa a nivel del discurso, el género y el intertexto.

De acuerdo con estudios enfocados en el discurso deportivo, la metonimia resulta, junto con la metáfora, una de las figuras retóricas más asiduamente explotada por los periodistas deportivos (Saiz Noeda, 2010; Quintero Ramírez y Álvarez Amaral, 2016; Suárez Ramírez y Suárez Muñoz, 2016).

De acuerdo con nuestros antecedentes, las metonimias más recurrentes en el discurso deportivo son aquellas que refieren al atleta o al equipo por su ciudadanía o nacionalidad (Medina Cano, 2010: 202), por los colores del uniforme que viste (Medina Cano, 2010: 202; Nomdedeu Rull, 2004: 85) y por alguna otra característica relevante que lo distingue de otros deportistas o equipos (Medina Cano, 2010: 202; Quintero Ramírez, 2013: 86). También son comunes las metonimias que hacen referencia a un deportista por el número que este lleva en alguna parte del uniforme (Medina Cano, 2010: 202).

Asimismo, de acuerdo con Suárez Ramírez (2015: 208), otras metonimias asiduamente utilizadas en el texto deportivo son aquellas que mencionan la ciudad o el país que representa un equipo por el equipo en sí, así como aquellas que nombran el instrumento de una actividad que realiza el atleta para aludir a este último. Nomdedeu Rull (2004: 151) añade que, en el caso de algunos deportes de conjunto, los atletas suelen nombrarse por la demarcación que juegan. Por último, Quintero Ramírez (2013: 92) advierte que también se designa a un atleta por algún logro que ha alcanzado en su carrera deportiva.

\section{METODOLOGÍA}

\subsection{Configuración del corpus}

"Llevar a cabo un estudio sobre el léxico del lenguaje del deporte es harto complicado, ya que aparte de que este campo genera una cantidad enorme de términos y unidades fraseológicas, hay que tener en cuenta si este análisis habría que realizarlo basándose en los medios orales, escritos, audiovisuales, etc.” (Medina Montero, 2007: 197).

Con base en las observaciones de Medina Montero (2007), así como en el hecho de que hemos llevado a cabo estudios anteriores respecto de figuras retóricas en el marco de textos hablados, más específicamente de narraciones deportivas, en el presente estudio decidimos conformar un corpus de notas periodísticas de la prensa electrónica a fin de establecer parangones con los antecedentes. 
Además, consideramos que en el ámbito escrito puede manifestarse más claramente la función textual de las metonimias, ya que se trata de un medio que implica mayor elaboración y cuidado. "La prensa invita al lector a abandonar su papel de fanático para ocupar el lugar del conocedor, del experto y hacer razonable el resultado" (Medina Cano, 2010: 174). Asimismo, es el espacio de la prensa donde se producen más metonimias que metáforas (Cf. Quintero Ramírez y Álvarez Amaral, 2016).

De esta manera, el corpus está constituido de 150 notas de la versión en línea de diferentes diarios en español, francés e inglés; esto es, 50 notas por cada lengua. Los diarios de los que obtuvimos las notas deportivas en español son: La Jornada, Excélsior y Esto de México; La Vanguardia, El Mundo y El País de España; Clarín de Argentina; El Comercio de Perú; El Heraldo de Colombia y El Nacional de Venezuela.

Las notas periodísticas en francés se extrajeron de los siguientes diarios: Le Soir, L'Avenir y Metro de Bélgica; L'Essentiel de Luxemburgo y La Croix, 20 Minutes, Cnews matin, Ouest-France, Le Monde y L'Équipe de Francia.

Finalmente, las notas en inglés fueron tomadas de los siguientes periódicos: Herald Sun de Australia; Toronto Star de Canadá, Hindustan Times y Times of India de India; Trinidad Express de Trinidad y Tobago; The New Zealand Herald de Nueva Zelanda; The Guardian UK y The Daily Star de Gran Bretaña y Daily News y LA Times de Estados Unidos.

Las notas periodísticas deportivas consideradas para el corpus fueron publicadas en sus respectivos diarios durante los meses de junio y julio de 2017. Dichas notas tratan una variedad de deportes tales como: fútbol, tenis, automovilismo, pugilismo, rugby, béisbol, baloncesto, ciclismo, entre otros. En la tabla 1 se aprecia la clasificación de las notas periodísticas de cada corpus de acuerdo con el tipo de deporte del que trata cada una de ellas.

\begin{tabular}{|l|c|c|c|}
\hline & Español & Francés & Inglés \\
\hline Fútbol & 14 & 11 & 10 \\
\hline Tenis & 9 & 9 & 6 \\
\hline Automovilismo & 2 & 4 & 2 \\
\hline Baloncesto & 4 & 5 & 5 \\
\hline Hipismo & 1 & 1 & 3 \\
\hline Boxeo & 2 & 2 & 3 \\
\hline Atletismo & 2 & 2 & 3 \\
\hline Béisbol & 6 & - & 6 \\
\hline Ciclismo & 1 & 7 & - \\
\hline Fútbol americano & 2 & - & 3 \\
\hline Clavados & 2 & - & - \\
\hline Toreo & 2 & - & - \\
\hline Lucha libre & 2 & - & - \\
\hline Rugby & - & 3 & 2 \\
\hline Handball & - & 3 & - \\
\hline Golf & - & 1 & 1 \\
\hline Alpinismo & - & 1 & - \\
\hline Críquet & - & - & 4 \\
\hline Vóleibol & - & - & 1 \\
\hline Otros menesteres & 1 & 1 & 1 \\
\hline
\end{tabular}

Tabla 1. Clasificación de las notas periodísticas por deportes. 
Como pretendemos llevar a cabo un análisis contrastivo y nos planteamos el objetivo de identificar las metonimias más comunes en un corpus en español, francés e inglés, nos pareció más pertinente e interesante construir dicho corpus con base en notas de diarios de diferentes países hispanohablantes, francófonos y anglófonos. De tal manera, presentamos resultados más abarcadores del fenómeno en cuestión.

\subsection{Identificación y tratamiento de los resultados}

Luego de constituir el corpus, nos propusimos analizarlo exhaustivamente para alcanzar los objetivos que nos planteamos en el estudio. Así pues, en primer lugar, examinamos minuciosamente todas las notas a fin de identificar tanto las metonimias empleadas como sus respectivos referentes.

Una vez registradas las metonimias en cada lengua, observamos las características de aquellas que se repiten en las tres, así como los rasgos de las figuras que solamente se producen en una o dos de ellas. Con base en estos resultados, establecemos semejanzas y diferencias de las metonimias utilizadas por periodistas deportivos en los tres idiomas.

Por último, inspeccionamos las notas periodísticas completas con la intención de reconocer la función que despliegan estas figuras retóricas a nivel del texto. Los resultados que obtuvimos de nuestro corpus los contrastamos con aquellos de estudios previos a fin de establecer nuestras conclusiones.

\section{RESULTADOS Y ANÁLISIS}

En este apartado presentamos, en primer lugar, las metonimias que resultan comunes en las tres lenguas que nos ocupan en el marco de nuestro corpus. En segundo lugar, presentamos aquellas metonimias que resultan exclusivas de una de las lenguas. Para ilustrar dichas figuras retóricas, consideramos fragmentos textuales a fin de evidenciar no solamente los rasgos de la metonimia aludida, sino también el referente respectivo.

\subsection{Metonimias comunes en las tres lenguas}

Una de las metonimias más usuales en las tres lenguas del corpus son las que se refieren a un atleta por medio de su nacionalidad, tal como lo advierte Medina Cano (2010: 202), como observamos en los ejemplos (1-3).

(1) Quien sí lo reconoce es su sucesor, Claudio Borghi. El argentino fue contundente y le dio todos los méritos al 'Loco'. [El Comercio - 30/06/2017]

(2) Rafael Nadal, légende de la terre battue à Roland-Garros L'Espagnol a écrit une nouvelle page de sa légende en remportant, dimanche 11 juin, son dixième titre sur la terre battue de Roland-Garros. [La Croix - 11/06/2017]

(3) For Ricciardo, on his team's 'home' Red Bull Ring circuit, it was a fifth consecutive podium finish and the 22nd of his career. The Australian smiled: "It was a fun race with some decisive moments at the start and then defending Lewis in the last laps. It got pretty close!" [...]. [Herald Sun - 09/07/2017] 
Otras metonimias recurrentes, como lo señalan en sus respectivos estudios Nomdedeu Rull (2004: 84) y Quintero Ramírez (2013: 92), son aquellas que se refieren a un deportista por la posición que juega en el terreno de juego, como en los ejemplos (4-6).

(4) El cuarto fue obra de Neymar, su gran valedor. "Paulinho es un amigo que tengo en el fútbol. Quiero que fiche por el Barça porque con un jugador como él, que es un amigo también, nosotros vamos a ganar mucho", ha dicho el delantero. [La Vanguardia 10/07/2017]

(5) Antoine Rabillard quitte Marseille. Le contrat du joueur de 21 ans a été résilié un an avant son terme. La saison dernière, l'attaquant a fait deux apparitions en Ligue 1. [L'Équipe 24/07/2017]

(6) Alexandre Lacazette, Lyon

The striker appeared to be bound for Atlético Madrid before the court of arbitration for sport upheld the Spanish club's transfer ban, opening the door for Arsenal. [The Guardian $-21 / 06 / 2017]$

También hemos encontrado metonimias que remiten a un atleta por el club deportivo para el que trabaja, este es el caso de los ejemplos siguientes (7-9), en los que la figura retórica explota el recurso del sustantivo común jugador/ joueur/ player y la precisión del club deportivo. En español y francés dicha precisión se evidencia a través de un sintagma preposicional (del Real Madrid y de Burnley respectivamente); mientras que en inglés la especificación se resuelve antecediendo el sustantivo propio Chelsea al sustantivo común player. Cabe señalar que en los tres idiomas el determinante definido encabeza el sintagma en cuestión ( $e l$, le y the respectivamente).

(7) Cristiano Ronaldo había elegido ser último y no pudo hacer nada [...] En un video de YouTube se aprecian los duros comentarios de un grupo de periodistas mexicanos de ESPN que no soportaron que el jugador del Real Madrid no patee primero. [El Comercio $-29 / 06 / 2017]$

(8) La décision intervient quelques semaines après la suspension fin avril par la FA pour 18 mois du controversé Joey Barton pour près de 1.260 paris sur des matchs entre 2006 et 2016. Le joueur de Burnley avait alors critiqué la FA [...] [20 Minutes - 22/06/2017]

(9) Origi was not in the game much after a couple of early hints of promise but more worrying for Belgium was the fact that Hazard was also a peripheral figure. When the Chelsea player did receive the ball he generally looked to bring it inside and make space for a shot [...]. [The Guardian - 01/07/2017]

En muchas ocasiones un atleta es nombrado simplemente por el oficio que profesa, como en (10-12), donde las metonimias el jugador, la boxeuse y the player retoman a sus respectivos referentes en el texto. Si bien en los ejemplos anteriores el periodista recurre a metonimias simples, consideramos que en dichas notas es claro e inequívoco el referente en cuestión, pues desde el titular se establece cuál será el hilo conductor de la nota. En efecto, en (10), el titular del diario es: Paulinho espera que el Barça le rescate de la liga China; en (11) se lee el siguiente titular: Sarah Ourahmoune, la combattante; y en (12) el titular se despliega así: Transfer roundup: Liverpool close to signing Andrew Robertson from Hull. 
(10) La incorporación de Paulinho al Barcelona sería un hecho verdaderamente remarcable [...] Hace cuatro años de aquella operación, pero el jugador no consiguió triunfar en la Premier y al cabo de dos temporadas emprendió una nueva aventura exótica, la que le llevó a Guanzhou. [La Vanguardia - 10/07/2017]

(11) Lorsqu'en 1998 les combats de boxe féminine sont autorisés, Sarah Ourahmoune les enchaîne malgré les sarcasmes de certains spectateurs qui lui conseillent " de retourner dans sa cuisine ». Elle va rafler dans sa carrière dix titres de championne de France. Pourtant, la boxeuse n'a jamais été épargnée. [Le Monde - 17/06/2017]

(12) Robertson joined Hull from Dundee United for $£ 2.85 \mathrm{~m}$ July in 2014 and despite the club’s struggles during that period, including most recently relegation from the Premier League, the player has regularly impressed. [The Guardian - 14/07/2017]

Asimismo, encontramos metonimias que aluden al equipo que representa a un país por el nombre de dicho país, tal como lo advierte Suárez Ramírez (2015: 208). Ejemplo de dichas metonimias son (13-15).

(13) México no pudo hacer nada contra Alemania y perdió 4-1 en las semifinales de la Copa Confederaciones. [El Comercio - 29/06/2017]

(14) Le Portugal de Cristiano Ronaldo a été neutralisé (2-2) par le Mexique, dimanche en Russie, avec pour la première fois l'incidence de la vidéo dans un tournoi international. [Le Monde - 08/06/2017]

(15) That tour was also notable for Ben Stokes's remarkable innings of 258- the quickest 250 in Test history and the highest score by a No6 batsman - and Kagiso Rabada's 13 wickets for 144 runs in South Africa's 280 run victory over England in the final Test. [The Guardian - 06/07/2017]

Igualmente, es común encontrar la alusión a un jugador o incluso a un caballo, como en el caso de (18) en el marco de los deportes hípicos, a través de una característica distintiva de este que es conocida por la afición deportiva. Lo anterior se evidencia en los ejemplos (16-18).

(16) Cristiano Ronaldo no pateó ningún penal en las semifinales ante Chile [...] La estrella portuguesa no pudo patear ningún penal porque antes el combinado luso había fallado sus tres primeros disparos y los chilenos anotaron todos. [El Comercio - 29/06/2017]

(17) Le Portugal, avec un Cristiano Ronaldo version altruiste auteur d'une passe décisive, a été neutralisé par le Mexique (2-2) en Coupe des confédérations [...] Mercredi, les champions d'Europe affronteront la Russie, leader du groupe A après avoir défait la Nouvelle-Zélande 2-0 samedi en ouverture du tournoi, tandis que les Mexicains rencontreront ces Océaniens. [Le Monde - 18/06/2017]

(18) Royal Symphony put in a "hang on to your hat" performance at Flemington on Saturday [...] The unbeaten colt, plucked from a paddock by Dunn and his wife Amanda as a yearling, is now a clear Caulfield Guineas favourite after streeting his rivals in the Taj Rossi Series Final. [Herald Sun - 08/07/2017]

También encontramos en el corpus de los tres idiomas metonimias que designan a un equipo por el color de la camiseta de su uniforme, como lo señalan Medina Cano (2010: 202) y Nomdedeu Rull (2004: 85), tal es el caso de (19-21) donde la selección nacional de fútbol de Chile es referida como la Roja, el club de fútbol de Liverpool es aludido como the Reds y la selección de rugby de Francia es denominada les Bleus. 
(19) Chile genera admiración

Ya en la última Copa del Mundo la Roja dio luces de lo que se venía al dejar fuera del certamen a la España multicampeona. [El Comercio - 30/06/2017]

(20) Virgil van Dijk has told Southampton the only move he wants this summer is to Liverpool. The Reds were forced to abandon their pursuit of the player after being accused of tapping him up. [Daily Star - 09/07/2017]

(21) Rugby : Le $\boldsymbol{X V}$ de France s'incline (37-15) face à l'Afrique du Sud.

Deux routes s'offraient aux Bleus, samedi à Durban, la victoire du rachat ou la défaite, synonyme de crise plus ou moins profonde en fonction de l'ampleur. [Le Monde - 17/06/2017]

Por último, y de manera menos recurrente, observamos que se alude a un atleta por alguna hazaña o logro conseguido en su carrera deportiva, como lo afirma Quintero Ramírez (2013: 92). Ejemplos de dichas metonimias se advierten en los ejemplos (22-24).

(22) Roger Federer sigue batiendo récords en Wimbledon [...] Sólo 42 minutos de juego, suficientes sin embargo para demostrar que el reciente vencedor del ATP 500 de Halle ha llegado en plena forma: intocable con el saque, 10 'aces', y dominando cada rincón de la 'Catedral'. [El Comercio - 04/07/2017]

(23) Le prochain feuilleton du duel entre Lewis Hamilton et Sebastian Vettel se tiendra, ce dimanche 25 juin, au Grand-Prix d'Azerbaïdjan à Bakou [...] Car il ne faut jamais enterrer le triple champion du monde et Mercedes trop tôt. [Cnews matin - 22/06/2017]

(24) Ronaldo, 32, has been summoned to appear before a judge near Madrid on July 31 to answer four counts of evading 14.7 million euros (\$16.8 million) in taxes. The four-time Ballon d'Or winner has protested his innocence with O Globo saying he had threatened to leave Real over the investigation. [The Times of India - 25/07/2017]

Por último, un fenómeno metonímico común en las notas analizadas en las tres lenguas es la alusión a deportistas mediante una combinación de lo que hemos señalado anteriormente. Ejemplos de esto se ilustran en (25-27). En efecto, en (25) Álvaro Morata es designado mediante su demarcación en la cancha y su nacionalidad. Igualmente, en (26) John McEnroe es denominado por el logro en su carrera y su nacionalidad. Finalmente, en (27) Juan Martín del Potro es referido en la nota por su ciudadanía y por un rasgo que lo distingue, este consiste en su ubicación en el ranking de la Asociación de Tenistas Profesionales (ATP) y en la preclasificación para un torneo específico, el campeonato de Wimbledon 2017.

(25) After Manchester United's pursuit of Antoine Griezmann ran into a brick wall, their search for a replacement for Zlatan Ibrahimovic took them to Real Madrid's Álvaro Morata. The Spanish striker remains top of their list but United have also been keeping an eye on a young Italian who looks destined to enjoy a wonderful career. [The Guardian 21/06/2017]

(26) Dans une interview au Sunday Times, John McEnroe a affirmé que Murray «fait toujours partie du Top 4 mondial, mais loin derrière». «Il l'est toujours. C'est surprenant qu'il soit N.1» mondial, a notamment affirmé le champion américain en faisant allusion au fait que Murray n'a gagné qu'un titre (à Dubaï) en 2017. [L'Avenir - 18/06/2017]

(27) Un cuadro complicado desde el comienzo espera a Juan Martín Del Potro en Wimbledon 2017. El tandilense, $32^{\circ}$ del mundo y preclasificado número 29 en el Grand Slam inglés, chocará en su primer partido con el australiano Thanasi Kokkinakis, que viene de obtener una victoria resonante en Queen's. [Clarín - 30/06/2017] 


\subsection{Metonimias exclusivas de una lengua del corpus}

Tras examinar el corpus, discurrimos que la mayoría de las estrategias metonímicas utilizadas son básicamente las mismas en las tres lenguas. No obstante, hay algunas metonimias que hemos identificado únicamente en una de las lenguas estudiadas. En efecto, un fenómeno que observamos solamente en inglés es la denominación de un jugador únicamente por la edad que este tiene, como es el caso de los ejemplos (28) y (29).

(28) As Wimbledon's absent queen discovered during that impertinent assault on her rule over Centre Court, Watson is a major threat when she is in the zone [...] Having fallen short with another attempt to reach the last 16 of a grand slam, the 25-year-old was hurting long after losing to Azarenka on Centre Court. [The Guardian - 07/07/2017]

(29) Murray went into the fortnight with doubts over his fitness and his form but he faces Italian Fabio Fognini today having breezed through his opening two matches [...] Back in April, when the 30-year-old admitted he only had a few years left at the top, there were those who reckoned he was on the way down after admitting to being in a "terrible" place before the French Open. [The Dailystar - 07/07/2017]

Si bien en las notas en español y francés se registran figuras retóricas que aluden a la edad de un deportista, dicho rasgo no es el único utilizado en la estrategia metonímica, como puede constatarse en (30) y (31). Ciertamente, además de dicho rasgo, se advierte la posición que juega el futbolista Paulinho en el terreno de juego en (30) y la nacionalidad de la tenista Mladenovic en (31).

(30) Paulinho espera que el Barça le rescate de la liga China

El centrocampista de 29 años no quiere encerrarse en la exótica jaula de oro en la que se han recluido tantas viejas glorias. [La Vanguardia - 10/07/2017]

(31) L'aventure de Kristina Mladenovic sur la terre battue parisienne s'est arrêtée en quart de finale. La Française de 24 ans s'est inclinée, mardi 6 juin, contre la Suissesse Timea Bacsinszky, en deux sets (6-4, 6-4). [07/06/2017]

En el caso de las notas en francés, observamos que suele utilizarse la metonimia que designa a un equipo por medio de sus miembros, como en (32), donde el equipo de handball de Nantes es denominado a través del sustantivo común genérico hommes precedido de determinante definido y seguido de un sintagma preposicional (de + nombre propio) que le confiere la especificidad a dicha metonimia y hace inequívoca la referencia ${ }^{2}$.

(32) Le HBC Nantes en sait un peu plus sur ses futures adversaires, en Ligue des champions, la saison prochaine. Hier, l'EHF a dévoilé la composition des pots, qui serviront de base au tirage au sort des poules, vendredi soir. Les hommes de Thierry Anti ne pourront pas rencontrer les Allemands de Kiel [...] [Ouest-France - 27/06/2017]

2 En un estudio anterior (Quintero Ramírez, 2016: 257) identificamos ejemplos de esta estrategia metonímica en español: “El '10' de la albiceleste brilló más que Cristiano, que se mostró poco asociativo y estuvo intermitente en la primera mitad, gracias al buen trabajo defensivo de los hombres de Gerardo 'Tata' Martino”. 
En español, en el caso de las notas beisboleras, observamos que se recurre a la designación del instrumento de una actividad que realizan los beisbolistas para hacer referencia a un equipo (Suárez Ramírez, 2015: 208), como se advierte en (33).

(33) El escudo real volvió a batirse con fuerza este domingo. El Rey se montó en la lomita, frente a su corte en pleno, para imponer un yugo en los bates de los Atléticos de Oakland. Con el primer pasado por las armas, llegó a los 2.303 de por vida y se dio la mano con el dominicano Juan Marichal, miembro de Cooperstown, en el quinto puesto histórico entre los lanzadores latinos. [El Nacional - 10/07/2017]

Otro fenómeno metonímico en español que únicamente encontramos en notas periodísticas de diarios latinoamericanos consiste en llamar paleador al beisbolista que batea de manera contundente. En (34) se emplea este recurso para aludir a Melky Cabrera. Dicha metonimia está fundamentada en la metáfora de asemejar el bate a una pala y equiparar el manejo de ambos instrumentos.

(34) Un sencillo del dominicano Melky Cabrera acabó con el sueño del novato Kyle Freeland de lanzar un no hit no run. El paleador de los Medias Blancas de Chicago pegó el batazo en el noveno inning, con un out, y sacó al lanzador del montículo. [El Nacional $10 / 07 / 2017]$

Si bien Nomdedeu Rull (2004: 151) señala que en su estudio registra algunas metonimias que designan una falta por el nombre del lugar donde esta se comete (penal, fuera de banda, córner, entre otras), así como metonimias que refieren a un objeto por una característica de este (por ejemplo: esférico o cuero para referir a la pelota), en el presente estudio dichas figuras resultan escasas. Por consiguiente, decidimos no dedicarles nuestra atención.

No obstante, tanto en francés como en español hemos encontrado en el marco de eventos tenísticos la referencia a una competencia por medio de un sustantivo que da cuenta del tipo de terreno en el que se juegan los partidos y un adjetivo gentilicio que denota la ciudad en donde tiene lugar dicho torneo. En efecto, en el ejemplo (35) el Campeonato de Wimbledon es designado como la hierba londinense y en (36) el Campeonato de Roland-Garros es denominado la terre battue parisienne.

(35) La española está acompañada en Wimbledon por su compatriota Conchita Martínez, campeona en la hierba londinense en 1994 y capitana actualmente de los equipos españoles de Copa Davis y Copa Fed. [La Voz de Galicia - 04/07/2016]

(36) Kristina Mladenovic a été éliminée, mardi 6 juin, en quart de finale de Roland-Garros [...] L'aventure de Kristina Mladenovic sur la terre battue parisienne s'est arrêtée en quart de finale. [La Croix - 11/06/2017]

\subsection{Función textual de las metonimias}

Ciertamente, como se ha mencionado en estudios anteriores, las figuras retóricas utilizadas en las notas deportivas ostentan la creatividad e inventiva que poseen los periodistas a fin de atraer al público lector (Nomdedeu Rull, 2004: 80; Quintero Ramírez, 2013: 83). No obstante, si nos centramos en la metonimia, esta no solamente hace alarde de dicha 
inventiva por parte del periodista, por el manejo de variantes denominativas para un mismo referente, sino también revela su capacidad para cohesionar el texto que redacta.

En efecto, percibimos que la metonimia despliega una función cohesionadora del discurso, pues, por los ejemplos que hemos apreciado a lo largo de esta sección, advertimos que el periodista deportivo recurre con insistencia a esta figura retórica a manera de anáfora a fin de aludir al referente que ha sido nombrado con anterioridad (Quintero Ramírez y Álvarez Amaral, 2016: 88). De esta manera, el reportero logra evitar repeticiones que puedan resultar tediosas y aburridas para el lector.

Para Denroche (2012), la deixis es totalmente metonímica: "Deixis is metonymic because it allows speakers to refer to one entity using different frames, depending on the speaker's perspective with regard to space (this chair here vs that chair there), person (my timetable vs your timetable), time (this meeting now vs that meeting then), etc." (Denroche, 2012: 88).

En (37) advertimos ejemplos de una diversidad de metonimias utilizadas para designar al referente Rafael Nadal, que se anuncia justo en el titular del texto. Las estrategias metonímicas van desde referir al atleta a través de un rasgo general hasta aludir a este mediante características muy particulares. Los recursos metonímicos de los que se vale el periodista deportivo para hacer referencia del tenista son: nacionalidad, ciudadanía, profesión, edad, logros o hazañas, rasgos físicos distintivos y una combinación de estos. De esta manera, el referente es expresado un sinnúmero de ocasiones en el texto sin que llegue a ser monótono para el lector.

(37) Rafael Nadal, légende de la terre battue à Roland-Garros

L'Espagnol a écrit une nouvelle page de sa légende [...]

Le tennisman espagnol Rafael Nadal s'effondre de joie [...]

L'Espagnol au regard noir devient alors invincible [...]

Le "roi ", sacré pour la première fois en 2005 à l'âge de 19 ans, privé de sa couronne pendant deux ans, a retrouvé son trône et personne ne pouvait le priver de cette consécration. $[\ldots]$

Après une longue période perturbée par les blessures, le Majorquin de 31 ans décroche son quinzième titre en Grand Chelem [...]

Vexé d'être évincé de son piédestal, le souverain déchu est reparti en champagne [...]

En sept rencontres, le Majorquin n'a perdu que 35 jeux et pas un seul set ! [...]

L'homme aux biceps imposants ajoute un nouvel épisode à sa légende [...]

L'émotion du natif de Manacor, sur l'île de Majorque, invite à mesurer la somme d'efforts et de sacrifices pour demeurer le plus grand joueur sur terre battue de tous les temps. [La Croix - 11/06/2017]

En (38) apreciamos un ejemplo en inglés en el que se emplean una variedad de metonimias para denominar a Aaron Judge. El referente se presenta claramente en el titular de la nota a través de su nombre y apellido. Posteriormente, dicho referente se retoma mediante estrategias metonímicas como la posición que juega a nivel defensivo, la función que desempeña a la ofensiva, la edad del beisbolista y un abanico de rasgos sobresalientes tanto actuales como potenciales.

(38) The AL All-Star balloting isn't just false hype - Aaron Judge is for real

The rookie slugger's combination of continued production and mounting popularity is deservedly translating to the baseball equivalent of high approval ratings at the polling centers [...]

Only potential future Yankee outfield mate Bryce Harper of Washington [...] 
Indeed, Judge not began the day leading the majors with 18 home runs, but the 25-yearold has captivated baseball fans in the Bronx and throughout the country [...]

The rookie right fielder homered in his first career game at Fenway Park on April 26 [...] He most certainly will be a marquee attraction for MLB to promote in the Home Run Derby, especially if pitted against hometown slugger Giancarlo Stanton of the Marlins. [Daily News - 07/06/2017]

Asimismo, observamos que las metonimias empleadas por los periodistas deportivos del corpus en las tres lenguas no son utilizadas al azar, pues es menester del periodista redactar su nota asegurándose de que no haya confusión alguna respecto de a quién se alude mediante las metonimias de las que se sirve. De tal suerte, el lector logra seguir el hilo conductor del texto sin perder de vista los referentes de las diferentes metonimias empleadas.

Finalmente, en (39) observamos un ejemplo de nota periodística en español en la que desde el titular se deja claro el referente del texto en cuestión, este es el clavadista Orlando Duque. En la bajada se recurre a la metonimia del gentilicio para aludir al atleta por su nacionalidad. Posteriormente, se retoma el referente antecedido del gentilicio para dar inicio a la nota en la entrada. En el segundo párrafo, el periodista vuelve a recurrir a la metonimia, esta vez aquella que da cuenta de un rasgo distintivo del deportista. Tanto al final de dicho párrafo como al inicio del siguiente, la referencia se lleva a cabo mediante el apellido del atleta. En el siguiente párrafo la alusión se hace una vez más a través de metonimia, esta vez aludiendo a un logro o hazaña del clavadista en cuestión.

(39) Orlando Duque volvió a la victoria en el mundial de clavados El colombiano superó a los favoritos Steven Lobue y Gary Hunt.

El colombiano Orlando Duque recordó sus mejores épocas con una fantástica victoria en San Miguel, Portugal, superando a rivales mucho más jóvenes, en el que fue su primer triunfo desde 2013 en la Serie Mundial del Red Bull Cliff Diving, superando a los favoritos, el norteamericano Steven Lobue y el inglés Gary Hunt.

Bajo el sol del archipiélago portugués de las Azores, fue el atleta más experimentado de los que participaban allí el que consiguió su primera victoria tras 27 eventos, a la edad de 42 años Duque se subió a lo más alto del podio.

Tras no lograr ningún podio en 2016, Duque se quedó solo a 0.7 puntos del podio en Irlanda, y en Portugal logró una racha de cuatro saltos muy consistentes que lo dejaron con una puntuación final de 346.50 puntos.

El campeón del mundo de $\mathbf{2 0 0 9}$ superó a LoBue y al recordman del certamen, Gary Hunt, quien sufrió mucho en las rocas volcánicas del Océano Atlántico, después de que sus dos primeros saltos, el sábado, le dejasen último en la general. [Heraldo - 09/07/2017]

Después de examinar las metonimias del corpus de nuestro estudio, podemos determinar que esta figura retórica es un recurso anafórico mediante el cual el periodista estructura su nota deportiva ofreciendo al público lector diversa información respecto del referente que ha mencionado con anterioridad.

\section{CONCLUSIONES}

Con base en las observaciones que hemos realizado a lo largo del estudio, discurrimos que existe una gran variedad de atributos de los que dan cuenta las metonimias en las notas 
periodísticas deportivas. Algunas metonimias recurren a hiperónimos, como aquellas que se refieren a un atleta por medio de un sustantivo común genérico como jugador, joueur, player. Otras metonimias son muy específicas y ofrecen al lector información peculiar de un equipo o deportista, por ejemplo: l'homme aux biceps imposants (para designar a Rafael Nadal).

A manera de recapitulación, las metonimias que hemos identificado en las tres lenguas del corpus se pueden escindir en dos grandes apartados: aquellas que se refieren a un equipo y las que designan a un atleta. Las primeras podrían inventariarse de la siguiente manera: a) país o ciudad que representa dicho equipo; b) color distintivo del uniforme; c) logros deportivos; d) miembros integrantes y e) un instrumento de la actividad realizada por los integrantes del equipo. Las metonimias empleadas para nombrar a un atleta podrían sintetizarse así: a) nacionalidad o ciudadanía; b) profesión ${ }^{3}$; c) posición o demarcación en el terreno de juego; d) edad; e) rasgos distintivos; f) hazañas o logros; g) clasificación de acuerdo con instancias oficiales.

Ahora bien, las metonimias particulares a una o dos lenguas son escasas, aunque igualmente importantes de resaltar. En los diarios en inglés se emplea la metonimia que designa a un atleta por su edad únicamente. En francés se alude con frecuencia a un equipo por medio de un sintagma nominal constituido del sustantivo genérico hommes precedido de determinante definido y seguido de sintagma preposicional. En español se emplea el recurso metonímico de hablar del instrumento de una actividad que realizan los beisbolistas para hacer referencia a un equipo. Por último, tanto en español como en francés, para referir un campeonato de tenis se recurre al tipo de terreno en el que se lleva a cabo el evento.

En nuestro estudio, a diferencia de trabajos anteriores (Nomdedeu Rull, 2004; Suárez Ramírez, 2015; Quintero Ramírez y Álvarez Amaral, 2016), no solamente hemos querido analizar el tipo de metonimias explotadas por el periodista deportivo, sino también examinar el lugar que ocupan dichas figuras retóricas en la nota como recurso textual. En efecto, al observar las notas periodísticas completas y advertir la diversidad de estrategias metonímicas a las que recurre el periodista deportivo, hemos identificado una función textual cohesionadora, pues el periodista utiliza desde metonimias genéricas hasta sumamente particulares como estrategia anafórica para darle unidad a su texto. Esto hace que el público receptor tenga a bien leer toda la nota sin tedio.

Finalmente, consideramos que la originalidad del presente estudio yace en su enfoque en la metonimia en el marco del discurso deportivo, ya que hay pocos trabajos al respecto. Igualmente, argüimos que otro rasgo original de este artículo es que ha pretendido contrastar el fenómeno en cuestión en tres lenguas. No obstante, somos conscientes de que nuestro trabajo es solo una aproximación a este tema.

\section{Referencias bibliográficas}

Alvar Ezquerra, M. (2009). “El deporte y los términos deportivos”, En Hernán Gómez-Prieto, B. (ed.). Il linguagio dello sport la comunicazione e la scuola. Milán: Edizioni Universitarie de Lettere Economia Diritto, pp. 149-166.

3 Con frecuencia el sustantivo que presenta la profesión es seguido de un sintagma preposicional que da cuenta del club deportivo al que pertenece el deportista. Por lo general, es el sintagma preposicional el que le confiere a la metonimia una particularidad del referente. 
Arroyo Almaraz, I. y F. García García (2012). "El léxico deportivo de las crónicas periodísticas del Mundial de fútbol 2010 ganado por España en El Poema de Mío Cid", Historia y Comunicación Social, 17, pp. 317-341.

Castañón Rodríguez, J. (2012). "El lenguaje periodístico del deporte en el idioma español del siglo XXI", Historia y Comunicación Social, 17: pp. 343-358.

Corado Valenzuela, H. (2011). Análisis Retórico de los Signos Sonoros y Lingüísticos de la Narración Radiofónica de Futbol en Emisoras de Frecuencia Modulada. Tesis. Guatemala: Universidad de San Carlos de Guatemala.

Denroche, Ch. (2012). Metaphor, metonymy, language learning and translation. PhD thesis. England: University of London.

Denroche, Ch. (2015). Metonymy and Language: A New Theory of Linguistic Processing. New York/ London: Routledge.

Gómez Torrego, L. (2010). "Aspectos gramaticales del lenguaje del fútbol”, Monográficos MarcoELE: Lenguas de Especialidad y su Enseñanza, 11: pp. 132-149.

Hernando Cuadrado, L. A. (1994). "Comunicación y lenguaje en el periodismo escrito", Didáctica, 6 (1): pp. 145-159.

Herráez Pindado, A. J. (2004). "La metáfora en la formación del vocabulario del ciclismo en francés", Ibérica, 7: pp. 107-123.

Kövecses, Z. (2002). Metaphor: A practical introduction. Oxford: Oxford University Press.

Lakoff, G. \& M. Johnson (1980). Metaphors we live by. Chicago: University of Chicago Press.

Mapelli, G. (2004). "Locuciones del lenguaje del fútbol", Atti del XXI Congresso dell'AISPI, Letteratura della memoria. La memoria delle lingue: la didattica e lo studio delle lingue della penisola iberica in Italia. Messina: Lippolis, pp. 171-181.

Martínez, S. (coord.). (2010). Fútbol-espectáculo, Cultura y Sociedad. México, D.F.: Afínita.

Martínez Valle, M. (1997). Medios gráficos y técnicas periodísticas. Buenos Aires: Ediciones Macchi.

Medina Cano, F. (2010). "Los narradores deportivos y sus epopeyas cotidianas". En Martínez, S. (coord.). Fútbol-espectáculo, Cultura y Sociedad. México: Afínita, pp. 157-207.

Medina Montero, J. F. (2007). "La metáfora en el léxico futbolístico: el caso de los participantes en español, y sus posibles equivalentes en italiano". En Luque Toro, L. (coord.). Léxico español actual: Actas del I Congreso internacional de léxico español actual. Venecia: Università Ca'Foscari di Venezia, pp. 197-240.

Medina Montero, J. F. (2009). "La metáfora en el léxico futbolístico el caso de la actividad deportiva en español, y algunas propuestas de traducción al italiano". En Luque Toro, L. (coord.). Léxico español actual II. Venecia: Università Ca'Foscari di Venezia, pp.155-202.

Medina Montero, J. F. (2015). "La metáfora en el lenguaje futbolístico: el caso del tiempo, el lugar y los útiles deportivos en español, y propuestas de traducción al italiano", Rivista Internazionale di Tecnica della Traduzione, 17: pp. 137-155.

Nomdedeu Rull, A. (2004). Terminología del fútbol y los diccionarios: elaboración de un diccionario de especialidad para el gran público. Tesis doctoral. España: Universidad Autónoma de Barcelona.

Oliva Marañón, C. (2012). "Lenguaje deportivo y comunicación social: prototipo coetáneo de masas", Revista de Comunicación de la SEECI, 28 (2): pp. 11-29.

Quintero Ramírez, S. (2013). "Tropos en la crónica beisbolera en emisiones televisivas latinoamericanas", Journal of Linguistics and Language Teaching, 4 (2): pp. 83-99.

Quintero Ramírez, S. (2016). "Estructuras sintácticas y léxicas para designar los elementos del evento futbolístico", Literatura y Lingüística, 34: pp. 245-264.

Quintero Ramírez, S. y D. Álvarez Amaral (2016). "Figuras retóricas en notas periodísticas deportivas", Verbum et Lingua, 8: pp. 75-91.

Saiz Noeda, B. (2010). "Notas sobre la retórica del lenguaje futbolístico", Monográficos MarcoELE: Lenguas de Especialidad y su Enseñanza, 11: pp. 132-149. 
Sánchez Manzanares, M. C. (2008). "Precisiones conceptuales en la definición de la sinécdoque como tropo metonímico". Pragmalingüística, 15-16: pp. 200-214.

Segrave, J. O. (2000). "The sports metaphor in American cultural discourse", Culture, Sport, Society, 3 (1): pp. 48-60.

Segura Soto, G. A. (2009). "Prepara, apunta, dispara... fusila al portero, la metáfora bélica en el fútbol", Káñina, Revista de Artes y Letras, 33 Especial: pp. 67-74.

Silaški, N. (2009). "Topic-triggered metaphors in newspaper headlines". Professional communication and translation studies, 2 (1-2): pp. 59-66.

Smith, A. (2015). "Figuras literarias en los titulares de la prensa costarricense", Revista de Lenguas Modernas, 22: pp. 35-42.

Suárez Ramírez, S. (2015). Los titulares en los cibermedios deportivos. Principales figuras retóricas y su aplicación didáctica. Tesis doctoral. España: Universidad de Extremadura.

Suárez-Ramírez, S. y A. Suárez-Muñoz (2016). "La retórica del titular deportivo en la prensa española”, Documentación de las Ciencias de la Información, 39: pp. 83-118.

Vélez, B. (2010). “¿Ciudadanos o clientes? Fútbol-espectáculo y sociedad en Colombia”. En Martínez, S. (coord.). Fútbol-espectáculo, Cultura y Sociedad. México, D.F.: Afínita, pp. 209-223.

Yanes Mesa, R. (2006). "La crónica, un género del periodismo literario equidistante entre la información y la interpretación”, Espéculo. Revista de estudios literarios. Disponible en: http://www.ucm. es/info/especulo/numero32/cronica.html (consultado el 14 de junio de 2017). 